\title{
EXTRACTION AND MICROENCAPSULATION OF POLYPHENOLS FROM ORTHOSIPHON STAMINEUS LEAVES
}

\author{
S. F. Pang ${ }^{1}$, M. M. Yusoff ${ }^{2 *}$ and J. Gimbun ${ }^{* 1,3}$ \\ ${ }^{1}$ Faculty of Chemical and Natural Resources Engineering, \\ Universiti Malaysia Pahang, 26300 Gambang, Pahang, Malaysia \\ *Email: jolius@ump.edu.my \\ Phone: +6095492899; Fax: +6095492889 \\ ${ }^{2}$ Faculty of Industrial Science and Technology, \\ Universiti Malaysia Pahang, 26300 Gambang, Pahang, Malaysia \\ ${ }^{3}$ Center of Excellence for Advanced Research in Fluid Flow, \\ Universiti Malaysia Pahang, 26300 Gambang, Pahang, Malaysia
}

\begin{abstract}
This paper presents an extraction and microencapsulation study of polyphenols obtained from Orthosiphon stamineus leaves. Extraction using aqueous solvent enabled a simultaneous extraction of both hydrophilic and lipophilic phenolic compounds. Higher solid concentration leads to higher solution viscosity, bigger particle size, lower moisture content and a less dented surface, which may improve particle flowability. The results suggest that eupatorin and rosmarinic acid are more susceptible to thermal degradation than sinensetin during spray drying. The formulation of 1:9 whey protein to maltodextrin ratio was also found to be more effective, with $86.7 \%$ total flavonoids retention compared to other formulations, viz, 1:1 and 9:1.
\end{abstract}

Keywords: Extraction; microencapsulation; misai kucing; eupatorin; sinensetin; rosmarinic acid.

\section{INTRODUCTION}

Orthosiphon stamineus (vernacular name: 'misai kucing') is consumed widely as a herbal tea among the Southeast Asian population. O. stamineus is traditionally used in Malaysia for treatment of bladder inflammation, eruptive fever, oedema, hepatitis, jaundice, hypertension, diabetes mellitus, gout, rheumatism, diuretic problems and influenza [1]. Previous studies revealed that extract of $O$. stamineus contained many medically useful bioactive compounds such as terpenoids, polyphenols and sterols that provide diuretic [2], antidiabetic [3], antiangiogenic and antiproliferative properties [4]. $O$. stamineus contains many useful active components such as rosmarinic acid, sinensetin and eupatorin, which can be recovered through the extraction process. The yield of bioactive component in the extract is affected by the type of solvent used, extraction method and condition. Most of the previous work related to extraction of polyphenol from $O$. stamineus has used thermally intensive extraction such as maceration [5] and accelerated solvent extraction [6]. These methods are often timeconsuming, besides being affected by thermal degradation of polyphenols due to heat exposure over a prolonged period. Nowadays, better extraction methods of polyphenols have been developed, such as ultrasonic-assisted extraction (UAE) [7, 8]. Extraction is a mass transfer process involving solvent transport to the solid phase (inner transport), 
dissolution of the solutes (solubility) and release of solutes from the solid matrix to the bulk phase (external transport). The UAE technique reduces the inner and external mass transfer limitation and hence increases the yield of extraction. Zhang et al. [8], for instance, showed that ultrasonic waves can break the cell membranes, reducing control of inner mass transport. Similarly, the rapid temperature rise during MAE also breaks the plant cell wall. Therefore, extractions using MAE and UAE methods were chosen for this work. The solvent type plays an important role in ensuring successful extraction of bioactive compounds from plant material. The solvent diffuses into the solid plant material and solubilizes compounds with a similar polarity during extraction [9]. It is understood that phenolic compounds concentrations in extracts from the same plant material may vary according to the solvent used. A combined effect of different extraction methods (ME and UAE) and varying solvent polarity on the polyphenol extraction from $O$. stamineus has never been studied previously, and hence this is one of the aims of this work. In the present study, ultra-performance liquid chromatography (UPLC) was used for the determination of polyphenols from $O$. stamineus extracts. UPLC provides improved separation in a shorter analysis time of $O$. stamineus extracts without compromising peak capacity and sensitivity [10].

A powder-based product is desirable for convenience of consumption, besides having a longer shelf-life and ease of handling. Spray drying is a common method of producing powder, but it requires very high temperatures, which may adversely affect the quality of the product. Furthermore, the polyphenol from O. stamineus extracts is prone to thermal degradation $[11,12]$. Thermal degradation of other bioactive compounds such as vitamin E and vitamin A is well documented [13]. The thermal degradation is undesirable because the degraded product is of low nutritional value and consequently hampers the intention to produce a nutraceutical product. The microencapsulation technique via spray drying is an effective way to protect the polyphenols against thermal degradation. Microencapsulation is defined as a process in which small particles are enclosed by a coating, or embedded in a homogeneous or heterogeneous matrix by an encapsulating agent. The choice of wall material is one of the main concerns for the microencapsulation process. Common microencapsulation agents such as whey protein isolate (WPI) and maltodextrin are often used for herbalrelated products $[14,15]$. Many studies related to the microencapsulation of plant extracts using different wall materials have been reported $[14,15]$, but none on $O$. stamineus. Therefore, this work aims to minimise the degradation of bioactive compounds from $O$. stamineus extract during spray drying using a microencapsulation technique by encapsulating agents such as whey protein isolate and maltodextrin.

\section{MATERIALS AND METHODS}

\section{Chemicals and Plant Material}

HPLC-grade solvents such as acetonitrile (ACN) and methanol were purchased from Merck (Darmstadt, Germany) and trifluoroacetic acid [16] was purchased from Fisher Scientific (Leics., UK). Dimethyl sulfoxide (DMSO), standard of rosmarinic acid, eupatorin and sinensetin were obtained from Sigma Aldrich (St. Louis, MO). Lactosefree whey protein isolate powder was obtained from Ultimate Nutrition (Fleetwood, UK) with $99 \%$ of undenatured proteins, while maltodextrin DE10 was obtained from San Soon Seng Food Industries (Malaysia). Leaves were collected in Gambang, Pahang, Malaysia from a white-flowered $O$. stamineus similar to one that has been deposited at 
the Forest Research Institute, Malaysia (voucher no. ZAS1113). Freshly collected leaves were washed with deionised water and dried at $37{ }^{\circ} \mathrm{C}$ for 3 days before being crushed to powder. Prior to use, the powder was kept in an air-tight plastic bag in a desiccator at room temperature to prevent moisture absorption.

\section{Extraction of Phenolic Compounds}

The powdered plant material was weighed $(1 \mathrm{wt} . \%)$ and mixed with solvent in a $250 \mathrm{ml}$ sealed Erlenmeyer flask. UAE was carried out in an ultrasonic bath (CREST P1800D, US) at $45 \mathrm{kHz}$ for 90 minutes and the temperature was set at $40{ }^{\circ} \mathrm{C}$. Maceration was performed at $40{ }^{\circ} \mathrm{C}$ in a stirred vessel. The supernatant was then separated from the residue by vacuum filtration through a $0.45 \mu \mathrm{m}$ nylon membrane.

\section{Microencapsulation by Spray Drying}

The extracts were encapsulated by two types of wall material, which are whey protein isolate and maltodextrin DE10. The encapsulant concentration of $6.67 \%$ was set for all samples, where the optimum polyphenols retention can be achieved [10]. O. stamineus extract was mixed with either WPI, maltodextrin or a combination of both encapsulants at ratios of 1:9, 1:1 and 9:1. The solution was mixed by magnetic stirring at $40{ }^{\circ} \mathrm{C}$ for 30 minutes to obtain a homogeneous solution. The resultant solution was spray dried using a lab scale spray dryer (Lab Plant SD06A, UK) fitted with a $0.5 \mathrm{~mm}$ atomizer, and an air velocity of about $4.1 \mathrm{~m} / \mathrm{s}$ was set constant throughout the experiment. The inlet air temperature was set at $180{ }^{\circ} \mathrm{C}$ and maintained at $\pm 1^{\circ} \mathrm{C}$ by the proportional-integralderivative controller. Feed was metered into the dryer by means of a peristaltic pump at $407.1 \mathrm{ml} / \mathrm{hr}$. Similar set-ups were employed for all experiments to ensure a fair comparison. Dried powder samples were collected from a Schott bottle attached at the bottom of the cyclone separator.

\section{Analysis of Polyphenols Content}

The total solid content from $O$. stamineus extract was determined by evaporating the liquid from $5 \mathrm{ml}$ solution completely in an oven. Moisture content for all dried powder samples is determined using a moisture analyser, and the water content is subtracted during preparation of solution for UPLC analysis of polyphenol after spray drying. The same dry weight of solid (bioactive compounds) is set for the initial solution (extract) and after drying the solution to ensure a fair comparison of polyphenol retention. The predetermined amount of dried powder was dissolved in $60 \%$ aqueous methanol with the aid of a vortex mixer to ensure dissolution of less polar compounds. The stock solution of rosmarinic acid $(10 \mathrm{mg} / \mathrm{ml})$ was prepared in methanol, whereas eupatorin $(10 \mathrm{mg} / \mathrm{ml})$ and sinensetin $(5 \mathrm{mg} / \mathrm{ml})$ were dissolved in DMSO. The three analytical standards were further diluted until $0.08 \mu \mathrm{g} / \mathrm{ml}$ to develop an eight-point calibration curve. Qualitative and quantitative determinations of the $O$. stamineus extract's major constituents (rosmarinic acid, sinensetin and eupatorin) were performed on a Waters Acquity UPLC H-Class (Milford, MA) fitted with an Acquity UPLC HSS T3 column $(2.1 \times 75 \mathrm{~mm}, 1.8 \mu \mathrm{m})$ and an Acquity UPLC HSS T3 VanGuard column guard (2.1 x 5 $\mathrm{mm}, 1.8 \mu \mathrm{m})$. The UPLC system is equipped with a photodiode array detector and connected to a computer running Waters Empower 2 software. The mobile phase consists of solvent A: water: TFA (20:0.001; v/v) and solvent B: ACN:TFA (20:0.001; 
$\mathrm{v} / \mathrm{v}$ ) and the following gradient elution: $0-2.0 \mathrm{~min}, 26 \% \mathrm{~B} ; 2.0-3.9 \mathrm{~min}, 26-50 \% \mathrm{~B}$; 3.9-6.9 min, 50-95\% B and finally washing the column with $95 \% \mathrm{~B}$ for $0.6 \mathrm{~min}$ and reconditioning the column with $26 \% \mathrm{~B}$ isocratic for $1.4 \mathrm{~min}$. The temperature was maintained at room temperature $\left(24^{\circ} \mathrm{C}\right)$, with an injection volume of $2 \mu \mathrm{l}$ and flow rate of $0.17 \mathrm{ml} / \mathrm{min}$. The sample was filtered with a $0.2 \mu \mathrm{m}$ PES membrane filter before being injected into the UPLC system. The peaks for rosmarinic acid (3.10-3.30 min), sinensetin (5.50-5.60 min) and eupatorin (5.65-5.75 min) were detected at $340 \mathrm{~nm}$.

\section{Statistical Analysis}

Each experiment was repeated in triplicate. Analysis of variance (ANOVA) was performed by using the data analysis tools in Microsoft Excel 2010, and a least significant difference (LSD) test was used to compare the means with a confidence interval of $95 \%$.

\section{RESULTS AND DISCUSSION}

\section{UPLC Quantification of Polyphenols}

The bioactive components were identified by means of the retention time and UV spectra of the standard (rosmarinic acid, sinensetin and eupatorin). UV spectra for all three target components were found to be well matched with each standard compound. Active components were quantified by comparing the peak areas with the results of a calibration series using standards obtained from Sigma-Aldrich. The calibration curves of the studied phenolic compounds showed good linearity $\left(r^{2}>0.997\right)$ in the range of $0.08-250 \mu \mathrm{g} / \mathrm{L}$ concentration. The limit of detection was determined by setting the signal to noise ratio of 3:1. The UPLC method developed for the first time by Pang et al. [10] is capable of a fast and accurate qualitative and quantitative analysis of polyphenols from $O$. stamineus extract. The analysis time is less than 7 minutes, as shown in Figure 1, which is about three times faster than those of the other reported methods $[3,17]$. The UPLC chromatogram shown in Figure 1 suggests that the presence of maltodextrin or whey protein does not affect polyphenol separation in the HSS T3 column. Thus a similar method for determination of polyphenol content was employed throughout this work.

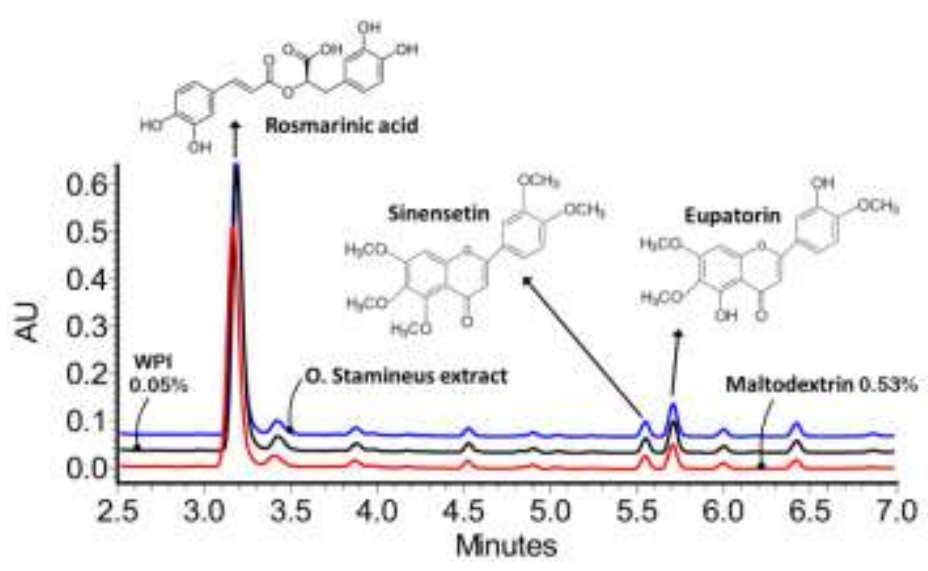

Figure 1. UPLC chromatogram for pure $O$. stamineus extract, spray dried powder using $0.05 \% \mathrm{WPI}$, and spray dried powder using $0.53 \%$ maltodextrin. 
Table 1. Effect of solvent type and extraction method on polyphenols extraction from $O$. stamineus.

\begin{tabular}{|c|c|c|c|}
\hline \multirow[t]{2}{*}{ Solvent type } & \multicolumn{3}{|c|}{ Bioactive component } \\
\hline & $\begin{array}{l}\text { Rosmarinic Acid } \\
\text { (mg RA/g DW) }\end{array}$ & $\begin{array}{l}\text { Sinensetin } \\
(\mu \mathrm{g} \mathrm{Sin} / \mathrm{g} \mathrm{DW})\end{array}$ & $\begin{array}{l}\text { Eupatorin } \\
\text { (mg Eup/g DW) }\end{array}$ \\
\hline \multicolumn{4}{|c|}{ UAE } \\
\hline Methanol & $33.13 \pm 0.19$ & $254.99 \pm 1.28$ & $1.87 \pm 0.01$ \\
\hline Isopropanol & $3.37 \pm 0.09$ & $261.21 \pm 1.01$ & $2.71 \pm 0.02$ \\
\hline Water & $0.245 \pm 0.09$ & $13.52 \pm 1.02$ & $0.04 \pm 0.06$ \\
\hline $50 \%$ Methanol & $34.84 \pm 0.002$ & $150.15 \pm 1.98$ & $0.45 \pm 0.05$ \\
\hline $70 \%$ Methanol & $38.70 \pm 0.06$ & $164.12 \pm 0.67$ & $0.98 \pm 0.01$ \\
\hline $50 \%$ Isopropanol & $35.33 \pm 0.05$ & $202.69 \pm 0.31$ & $1.57 \pm 0.01$ \\
\hline $70 \%$ Isopropanol & $36.91 \pm 0.12$ & $248.16 \pm 0.55$ & $2.38 \pm 0.03$ \\
\hline \multicolumn{4}{|c|}{ Maceration } \\
\hline $70 \%$ Isopropanol $2 \mathrm{~h}$ & $35.61 \pm 0.12$ & $210.25 \pm 3.48^{\mathrm{a}}$ & $2.17 \pm 0.02$ \\
\hline $70 \%$ Isopropanol $4 \mathrm{~h}$ & $36.35 \pm 0.26$ & $214.39 \pm 2.73^{\mathrm{a}}$ & $2.07 \pm 0.05$ \\
\hline
\end{tabular}

Note: Means (three or more replicates) followed by at least one same letter are not significantly different $(\mathrm{P}>0.05)$

\section{Influence of Solvent Type and Extraction Method on Polyphenols Extraction}

The influence of solvent type on polyphenols extraction was examined by employing solvents of various polarities $\left(\mathrm{MeOH}, \mathrm{IPA}, \mathrm{H}_{2} \mathrm{O}, 50 \% \mathrm{MeOH}, 70 \% \mathrm{MeOH}, 50 \%\right.$ IPA, $70 \%$ IPA) for both the ME and UAE methods. The extraction times for UAE and ME were set at 90 and 240 minutes respectively, following a preliminary study which indicated their optimum extraction time. Table 1 presented the effect of solvent type and extraction method on polyphenols extraction from $O$. No significant increases in extraction yield for ME were observed after 240 minutes. Meanwhile, prolonging UAE extraction beyond 90 minutes actually decreases the yield due to thermal degradation. The solubility of the bioactive component in different solvents is affected by its structural characteristics. Highly methoxylated compounds such as sinensetin and eupatorin, which are lipophilic, were more stable in a low polar solvent such as isopropanol. Similar findings are also reported by Akowuah et al. [5], who found that the amount of sinensetin and eupatorin is higher in a low polar solvent, i.e. chloroform extract. However, a highly hydroxylated compound such as rosmarinic acid is hydrophilic, and thus more soluble in methanol than in isopropanol. For the same reason, rosmarinic acid can be found in water extract, but not sinensetin and eupatorin. The results suggest that aqueous alcoholic solvent has a higher (> 20\%) extracting capacity of flavonoid and phenolic content compared to pure solvent, which is consistent with findings from other researchers [18]. Wach et al. [18], for instance, found that aqueous methanol ranging from 40 to $80 \%$ is preferable for rutin and quercetin extraction from $H$. perforatum. It is thought that aqueous solvent provides a wider range of polarity in contrast to pure solvent, and hence enhances simultaneous extraction of both methoxylated and hydroxylated compounds. This phenomenon can be seen clearly in the case of aqueous isopropanol, which increases extraction of rosmarinic acid (hydroxylated compound) more than tenfold from 2.93 to $34.78 \mathrm{mg} / \mathrm{g}$ DW without adversely affecting the extraction of the methoxylated flavonoid (sinensetin and eupatorin). 
Water is capable of extracting phenolic and flavonoid content using the UAE method. The concentrations of the three targeted compounds were barely detected from the UAE extract. In fact, the water extract sample has to be freeze-dried before the phenolic compounds can be deduced accurately from the UPLC analysis. Extraction of polyphenol using pure solvent (MeOH or IPA) fails to extract efficiently the highly hydroxylated compound (rosmarinic acid). Maceration which was performed only for the $70 \%$ aqueous isopropanol showed as good polyphenol extraction as the UAE. Nevertheless, the UAE method has the advantage of being faster at 90 minutes compared to 4 hours for the maceration technique.

\section{Microencapsulation of Polyphenolic Compounds from O. stamineus}

The polyphenols retention of encapsulated powder using either maltodextrin, whey protein or a combination of both substances was compared to the initial solution to assess the level of polyphenols preservation. The initial solution (denoted as 'extract' in Figure 2) and the dried powder were examined for their rosmarinic acid, sinensetin and eupatorin content using UPLC. All formulations of microencapsulant tested in this work showed a good retention (ranging from 65.39 to 82.08\%) of the targeted components in O. stamineus extract (Figure 2). The sample without microencapsulation which is indicated as 'control' in Figure 2 showed severe degradation of polyphenol content, especially for rosmarinic acid (54.24\%) and eupatorin (39.81\%). The retentions of individual polyphenol without microencapsulation are $45.76 \%, 78.31 \%$ and $60.19 \%$ for rosmarinic acid, sinensetin and eupatorin respectively. Both the rosmarinic acid and eupatorin showed higher degradation than sinensetin due to the presence of hydroxyl groups in their molecular structure. Flavonoid with more hydroxyl groups is known to be more susceptible to thermal degradation [19]. The absence of hydroxyl in the sinensetin's molecular structure explains its stability during spray drying, even without microencapsulation. Results from the UPLC analysis show that the sample encapsulated solely with whey protein provides an improvement of retention on all three targeted compounds, viz, rosmarinic acid (79.97\%), sinensetin (74.57\%) and eupatorin (65.39\%), as opposed to without encapsulation, as shown in Figure 2. Microencapsulation using pure maltodextrin provides even better polyphenol retention of rosmarinic acid $(82.67 \%)$, sinensetin $(82.24 \%)$ and eupatorin $(80.19 \%)$. The higher polyphenol retention by maltodextrin as opposed to WPI is attributed to loss of protein solubility during spray drying, which renders the entrapped polyphenols less accessible.

A blend containing WPI and maltodextrin has been employed successfully for microencapsulation of anhydrous milk fat [20] and conjugated linoleic acid [21]. Their finding implies that microencapsulation using a combination of protein and maltodextrin provides a much better accessibility to the encapsulated core, besides having better emulsifying properties, thus resulting in better microencapsulation efficiency. In this work, various formulations of microencapsulation agent were prepared, i.e., with WPI to maltodextrin ratios of 1:9, 1:1 and 9:1. The result shown in Figure 2 suggests a better retention of target component when a lower concentration of WPI is employed, i.e., with a WPI to maltodextrin ratio of $1: 9$, which shows retention of $89.41 \%$ of rosmarinic acid, $89.14 \%$ of sinensetin and $86.66 \%$ of eupatorin. These values are higher than those obtained when WPI or maltodextrin served as the sole wall material (see Figure 2). A previous study by Anandharamakrishnan et al. [22] suggests that increases in the total solid content cause a higher protein denaturation at temperatures above $100{ }^{\circ} \mathrm{C}$, which is often the case during spray drying. Higher 
concentration of WPI reduces the active component retention, which can be attributed to a higher percentage of protein denaturation during spray drying, which in turn causes a reduction in its solubility. Polyphenols entrapped inside the denatured protein may not be as accessible as those encapsulated by undenatured soluble protein, and hence may affect the release of polyphenols to the extracting solvent. Proteins are known to be an effective microencapsulating agent at very low concentrations compared with existing materials such as maltodextrin [21]. They reported up to $98 \%$ retention of conjugated linoleic acid when a WPI:maltodextrin ratio of 1:10 is employed, which is higher than those encapsulated solely by WPI (96.3\%) or maltodextrin (93.2\%). Earlier, Rosenberg and Sheu [23] successfully improved volatiles retention via microencapsulation using a formulation of WPI and lactose.

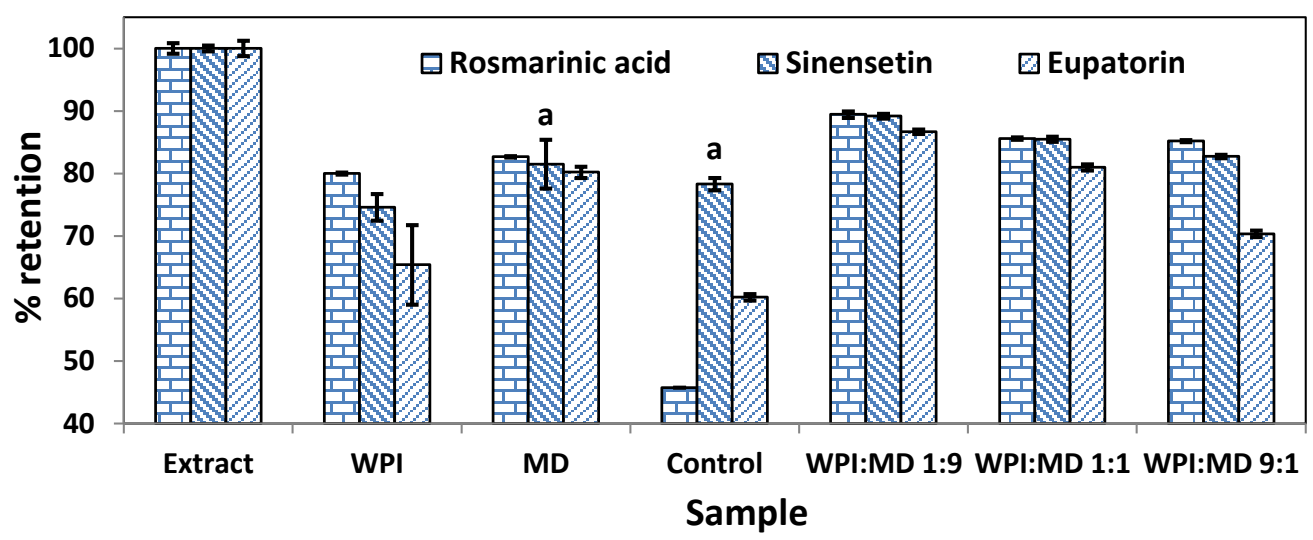

Figure 2. Retention of rosmarinic acid, sinensetin and eupatorin using different encapsulation strategies. Means of three replicates followed by at least one same letter are not significantly different $(\mathrm{P}>0.05)$.

\section{CONCLUSIONS}

The highest phenolic content of $96.41 \mathrm{mg} \mathrm{GAE} / \mathrm{g}$ DW was obtained using 50\% aqueous methanol, whereas the highest yield of rosmarinic acid (38.70 $\mathrm{mg} \mathrm{RA} / \mathrm{g} \mathrm{DW}$ ) was obtained using $70 \%$ aqueous methanol. The highest yield of sinensetin $(261.21 \mu \mathrm{g} \mathrm{Sin} / \mathrm{g}$ DW) and eupatorin (2.71 mg Eup/g DW) was obtained using isopropanol. Aqueous solvent provides a wider range of polarity as opposed to the pure solvent, and hence enhances simultaneous extraction of both methoxylated and hydroxylated compounds. The UAE method has the advantage of being faster, at 90 minutes compared to 4 hours for the maceration technique. Microencapsulation of polyphenols from O. stamineus using WPI, maltodextrin or a combination of both encapsulating agents has successfully reduced polyphenol degradation during spray drying. The highest polyphenol retention of rosmarinic acid $(89.41 \%)$, sinensetin $(89.14 \%)$ and eupatorin $(86.66 \%)$ was achieved by microencapsulation using a mixture of $1: 9$ protein to maltodextrin ratio. The results showed that both rosmarinic acid and eupatorin are more susceptible to thermal degradation than sinensetin during spray drying. 


\section{ACKNOWLEDGEMENTS}

Funding from the Ministry of Higher Education Malaysia (RACE RDU121308 and FRGS RDU130136) and Universiti Malaysia Pahang (GRS110352) is gratefully acknowledged.

\section{REFERENCES}

[1] Ho C-H, Noryati I, Sulaiman S-F, Rosma A. In vitro antibacterial and antioxidant activities of Orthosiphon stamineus Benth. extracts against foodborne bacteria. Food Chemistry. 2010;122:1168-72.

[2] Arafat O, Tham S, Sadikun A, Zhari I, Haughton P, Asmawi M. Studies on diuretic and hypouricemic effects of Orthosiphon stamineus methanol extracts in rats. Journal of Ethnopharmacology. 2008;118:354-60.

[3] Mohamed EAH, Yam MF, Ang LF, Mohamed AJ, Asmawi MZ. Antidiabetic Properties and Mechanism of Action of Orthosiphon stamineus Benth Bioactive Sub-fraction in Streptozotocin-induced Diabetic Rats. Journal of Acupuncture and Meridian Studies. 2013;6:31-40.

[4] Dolečková I, Rárová L, Grúz J, Vondrusová M, Strnad M, Kryštof V. Antiproliferative and antiangiogenic effects of flavone eupatorin, an active constituent of chloroform extract of Orthosiphon stamineus leaves. Fitoterapia. 2012;83:1000-7.

[5] Akowuah G, Ismail Z, Norhayati I, Sadikun A. The effects of different extraction solvents of varying polarities on polyphenols of Orthosiphon stamineus and evaluation of the free radical-scavenging activity. Food Chemistry. 2005;93:311-7.

[6] Pouralinazar F, Yunus MAC, Zahedi G. Pressurized liquid extraction of Orthosiphon stamineus oil: Experimental and modeling studies. The Journal of Supercritical Fluids. 2012;62:88-95.

[7] Tabaraki R, Nateghi A. Optimization of ultrasonic-assisted extraction of natural antioxidants from rice bran using response surface methodology. Ultrasonics Sonochemistry. 2011;18:1279-86.

[8] Zhang H-F, Yang X-H, Zhao L-D, Wang Y. Ultrasonic-assisted extraction of epimedin $\mathrm{C}$ from fresh leaves of Epimedium and extraction mechanism. Innovative Food Science \& Emerging Technologies. 2009;10:54-60.

[9] Ncube N, Afolayan A, Okoh A. Assessment techniques of antimicrobial properties of natural compounds of plant origin: current methods and future trends. African Journal of biotechnology. 2008;7:1797-806.

[10] Pang SF, Yusoff MM, Gimbun J. Assessment of phenolic compounds stability and retention during spray drying of Orthosiphon stamineus extracts. Food Hydrocolloids. 2014;37:159-65.

[11] Akowuah GA, Zhari I. Effect of extraction temperature on stability of major polyphenols and antioxidant activity of Orthosiphon stamineus leaf. Journal of herbs, spices \& medicinal plants. 2010;16:160-6.

[12] Al-Kayiem HH, Md Yunus Y. Drying of empty fruit bunches as wasted biomass by hybrid solar-thermal drying technique. Journal of Mechanical Engineering and Sciences. 2013;5:652-61. 
[13] Xie YL, Zhou HM, Liang XH, He BS, Han XX. Study on the morphology, particle size and thermal properties of vitamin A microencapsulated by starch octenylsucciniate. Agricultural Sciences in China. 2010;9:1058-64.

[14] Betz M, Steiner B, Schantz M, Oidtmann J, Mäder K, Richling E, et al. Antioxidant capacity of bilberry extract microencapsulated in whey protein hydrogels. Food Research International. 2012;47:51-7.

[15] Şahin-Nadeem H, Dinçer C, Torun M, Topuz A, Özdemir F. Influence of inlet air temperature and carrier material on the production of instant soluble sage (Salvia fruticosa Miller) by spray drying. LWT-Food Science and Technology. 2013;52:31-8.

[16] Farahany S, Bakhsheshi-Rad HR, Idris MH, Abdul Kadir MR, Lotfabadi AF, Ourdjini A. In-situ thermal analysis and macroscopical characterization of $\mathrm{Mg}-$ xCa and Mg-0.5Ca-xZn alloy systems. Thermochimica Acta. 2012;527:180-9.

[17] Pang S, Yusoff M, Gimbun J. Assessment on bioavailability of flavonoids from orthosiphon stamineus during spray drying. Developments in Sustainable Chemical and Bioprocess Technology. 2013:137-44.

[18] Wach A, Pyrzyńska K, Biesaga M. Quercetin content in some food and herbal samples. Food Chemistry. 2007;100:699-704.

[19] Buchner N, Krumbein A, Rohn S, Kroh LW. Effect of thermal processing on the flavonols rutin and quercetin. Rapid Communications in Mass Spectrometry. 2006;20:3229-35.

[20] Young S, Sarda X, Rosenberg M. Microencapsulating properties of whey proteins. 2. Combination of whey proteins with carbohydrates. Journal of Dairy Science. 1993;76:2878-85.

[21] Choi K-O, Ryu J, Kwak H-S, Ko S. Spray-dried conjugated linoleic acid encapsulated with Maillard reaction products of whey proteins and maltodextrin. Food Science and Biotechnology. 2010;19:957-65.

[22] Anandharamakrishnan C, Rielly CD, Stapley A. Loss of solubility of $\alpha$ lactalbumin and $\beta$-lactoglobulin during the spray drying of whey proteins. LWTFood Science and Technology. 2008;41:270-7.

[23] Rosenberg M, Sheu T. Microencapsulation of volatiles by spray-drying in whey protein-based wall systems. International Dairy Journal. 1996;6:273-84. 